\title{
The treatment of carcinoma of the lung by radiotherapy
}

\author{
ThOMAS J. DeEley \\ F.F.R., D.M.R.T.
}

\author{
Director, South Wales and Monmouthshire Radiotherapy Service
}

\begin{abstract}
Summary
Where the diagnosis of cancer of the lung is made by bronchoscopy the preferred treatment is surgery for squamous lesions and radiotherapy for anaplastic and oat-cell lesions. In inoperable tumours $8 \%$ of patients survive for more than 3 years after a radical course of radiotherapy; some clinical trials aimed at improving this survival rate are described. This lecture deals with the established disease, earlier diagnosis, before spread, would make radical treatment with a possibility of cure possible in more patients but this disease can be prevented to a large degree and this is the obvious method of attack.
\end{abstract}

THE possible ways of controlling cancer of the lung are: (1) to prevent it by removing the known causative factors; (2) to diagnose the disease at an early stage before dissemination has occurred so that local eradicative treatment can be given to the chest and (3) to improve existing methods or devise new methods to treat the established disease. Some progress has been made with the first two, we know of causative agents, the most important being tobacco smoking, but even if the advice of the medical profession was accepted by the public it would be many years before there was a marked reduction in the incidence of the disease. The high incidence of metastic spread in this disease accounts for its poor prognosis, but, even with very rapidly growing tumours, there is a time when the disease is localized to the chest and where local treatment may produce a cure. Until we can achieve prevention or attain early diagnosis the only alternative is to improve the existing methods of treatment and look for new techniques.

The methods available at present are surgery, radiotherapy, chemotherapy and various combinations of these. When the patient presents, a decision must be made of the form of treatment to be given; this could be radical aimed at a 'cure', palliative to relieve distressing symptoms, or, if neither of these is applicable-no active treatment; nothing will be achieved in giving a long, difficult or upsetting course of treatment to a patient who has widespread disease but with no symptoms requiring palliation.

\section{Radical treatment}

This could be either by surgery or radiotherapylet us deal with operable lesions first. A controlled clinical trial to compare radical surgery and radical radiotherapy in operable patients was reported by Morrison, Deeley \& Cleland (1963). Patients accepted to the trial were all assessed by a team of surgeons, radiotherapists, chest physicians and physiologists and if it was considered that the lesion was operable either surgery or radiotherapy was carried out, the treatment given being selected by a system of stratified randomization. The results are shown in Table 1.

TABle 1. Carcinoma of bronchus. Operable patients

\begin{tabular}{lcc}
\hline \multicolumn{1}{c}{ Treatment: } & Surgery & Radiotherapy \\
\hline No. of patients & 30 & 28 \\
Four-year survival rate & $23 \%$ & $7 \%$ \\
\hline
\end{tabular}

It will be seen that the 4-year survival rate is decidedly better for surgery than for radiotherapy. However, further analysis according to the histology of the tumour showed that the survival rate for squamous lesions was $30 \%$ for surgery and $6 \%$ for radiotherapy but for anaplastic tumours the results were about the same. A nationwide trial carried out by the Medical Research Council compared the survival rates for operable oat-cell tumours and showed that treatment by radiotherapy was preferable (Miller, Fox \& Tall, 1969). Thus we can conclude that for central lesions, where bronchoscopy has produced material for histological examination, the treatment giving the best results for squamous tumours is surgery, whilst radiotherapy is preferred for anaplastic and oat-cell tumours.

\section{Inoperable lesions}

Only about $10 \%$ of patients attending hospital with a carcinoma of the bronchus have a surgical eradication. In the remaining patients some will be suitable for radical radiotherapy if the tumour is still localized to the chest. Radiotherapy is a relatively young speciality and is still in the process of developing. X-rays were discovered by Roentgen 
only some 75 years ago, these rays were of low energy and it was impossible to give a radical course of radiation to deep seated tumours in the chest. Improvements in machine design with more powerful $\mathrm{X}$-ray beams made it possible to give adequate doses to very small bronchus lesions but it was not until the 1950s that it was possible to treat all chest lesions adequately by means of megavoltage apparatus; only in relatively recent years have these machines been universally available. During this time we have attempted to determine the optimum treatment conditions for tumours at this site. The only way that this can be achieved is by controlled clinical trials, but these take many years to reach a conclusion when applied to malignant disease. Essentially they compare two accepted methods of treatment in identical groups of patients and attempt to find out which technique gives the best results taking into account survival rate, morbidity and complications of treatment. Whilst it is conventional to compare 5-year survival rates, a 3-year rate is a satisfactory index for comparison in this disease. The graph (Fig. 1) shows the survival rate for 513 patients with an inoperable cancer of the lung compared with a similarly composed group of normal people each of the same age and sex as the bronchus group. From 3 years onwards the two lines are parallel, that is, the possibilities of dying from cancer of the lung is no greater at 3 years than is the possibility of dying for the normal population.

In all cases treated by radiotherapy, a volume which includes the primary lesion with a surrounding area of $2.0 \mathrm{~cm}$ of apparently uninvolved lung together with the whole mediastinum is raised to a radical tumour dose.

The results of treatment in 513 patients treated by radiotherapy for an inoperable carcinoma of the bronchus were at 3 years $-8 \%, 4$ years $-7 \%$ and 5 years $-6 \%$. The factors affecting the prognosis were histology (better for squamous lesions), site of the tumour (better for upper lobe lesions), the presence of mediastinal nodes and chronic bronchitis invariably resulted in a poor prognosis.

We are continually trying to improve the results of treatment and I will briefly describe the results obtained from a series of controlled clinical trials, further details will be found in another article (Deeley, 1971). In anaplastic lesions a controlled clinical trial was designed to compare two different dose levels given by the same method of fractionation over the same period of time. It was found that 3000 rads given in twenty treatments in 4 weeks gave better survival rates than 4000 rads given with the same factors. It appeared that the difference in survival was due to the increased incidence of radiation fibrosis in the group receiving the greater dose. A similar trial showed that 4000 rads were preferable to 5000 rads in squamous lesions. (Deeley, 1967.) It is conventional to treat daily, five times a week for 4 weeks. If possible I prefer my patients to be treated from their own homes where they have lived for some time with their own brand of infective organisms. If they are admitted to hospital there is a risk that they may be cross-infected by other organisms which in their poor state of health may be serious; the patients are usually happier, also, if they are at home. However, daily travelling to hospital for treatment can be very exhausting and a trial was designed to compare twice weekly treatment with daily treatment. To achieve the same biological effect the total tumour dose was reduced by $20 \%$. The trial showed that there was no difference in survival, morbidity or complications between the two groups and we now treat twice weekly.

To determine the effect of adjuvant therapy two similar groups of patients received the same radiotherapy to the chest, but one group had, in addition, chlorambucil with the idea of ablating small sub-

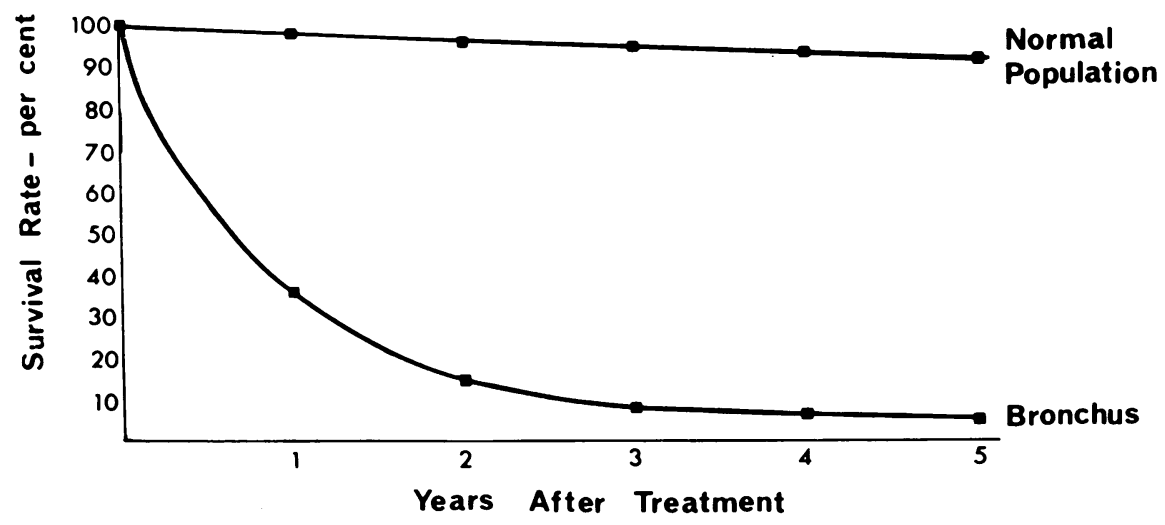

FIG. 1. Survival curve for patients treated for an inoperable carcinoma of the bronchus compared with survival curve for normal people of the same age and sex. 
clinical deposits. The results for the two groups were the same (Deeley, 1971). Further trials need to be carried out with other chemotherapeutic agents, either singly or in various combination of multiple drugs.

Injection of Synkavit (tetrasodium-2-methyl-1: 4naphtho-hydroquinone diphosphate) before treatment produced an added expectation of life of 2 months at 6 months, but at 2 years the results were the same for the two groups (Deeley, 1962).

Clinical trials where patients have been treated in hyperbaric oxygen have so far produced no increase in survival. Many workers are carrying out controlled clinical trials aimed at determining the optimal treatment conditions.

\section{Special cases}

There are a few special cases that I should mention (Deeley, 1967).

(a) Superior vena caval obstruction: The association of a poor prognosis with this syndrome has occurred because workers have grouped all cases of obstruction together in their reports irrespective of whether or not distant metastases were present. If there is no evidence of distant spread radical treatment is given and the results are the same as radical treatment for inoperable patients without obstruction. If distant metastases are present, palliative therapy is given to relieve symptoms.

(b) Pleural effusion: A blood-stained effusion means that the pleura is involved potentially over its whole area and is a contra-indication to treatment; a primary lesion with a serous effusion, however, should be treated radically.

(c) Tuberculosis: If there is active tuberculosis present in the chest the appropriate antitubercular drugs are given. There is no evidence that radiation activates a quiescent or healed lesion.

(d) Cerebral metastases: About one-quarter of cerebral deposits are 'solitary'-that is, there are no other deposits present in the body. If there are no clinical deposits demonstrable elsewhere we irradiate the whole brain and the chest to a radical tumour dose. In such patients $4 \%$ survived for more than 3 years, half the patients were able to return to a relatively normal life for at least 1 month and $7 \%$ for 1 year (Deeley \& Rice-Edwards, 1968).

\section{Effects of irradiation}

Radiotherapy is invariably blamed for all postradiation effects. The radiation will kill some tissues and repair will result in radiation fibrosis. Cells in both normal lung and in the malignant tumour will be killed. The aim of radiotherapy is to kill malignant tissues with as little damage as possible to the normal tissues. The irradiated lung is particularly prone to infection and broad spectrum antibiotics should be given as soon as symptoms develop.

\section{Palliation}

Palliative treatment should aim at relieving distressing symptoms with the minimum disturbance to the patient. Secondary bone deposits causing pain are well worth treatment, often this can be given in a single application. Haemoptysis is often a distressing symptom and can be relieved in a large proportion of patients.

\section{Conclusion}

The results of treatment of the established disease are indeed very disappointing. If the condition is operable a number of patients can be expected to survive, if it is inoperable but confined to the chest a few patients can be salvaged by radical radiotherapy. It is important that the appropriate treatment is given and that the chance of a cure, however small, is not jeopardized by inadequate or inappropriate therapy. This demands very careful co-operation between the chest physician, chest surgeon and radiotherapist. It is possible that ongoing research work may achieve some small improvement in the survival rate after radical radiotherapy. Dramatic improvements will not be made until some method of dealing with distant metastases has been found. The great tragedy is that this is one of the few cancers which we know could be prevented.

\section{References}

DeELey, T.J. (1962) A clinical trial of Synkavit in the treatment of carcinoma of the bronchus. British Journal of Cancer, 16, 387.

DeELEY, T.J. (1966) A clinical trial to compare two different tumour dose levels in the treatment of advanced carcinoma of the bronchus. Clinical Radiology, 17, 299.

DeELEY, T.J. (1967) Review article: The treatment of carcinoma of the bronchus. British Journal of Radiology, 40, 801.

DeELEY, T.J. \& RiCe-EdWARDS, J.M. (1968) Radiotherapy in the management of cerebral secondaries from bronchial carcinoma. Lancet, i, 1209.

DeELEY, T.J. (1971) Controlled clinical trials in the treatment of carcinoma of the bronchus. Proceedings of the Second Congress of the European Association of Radiology, Amsterdam. Excerpta Medica.

Miller, A.B., Fox, W. \& TAll, R. (1969) Five-year followup of the Medical Research Council's comparative trial of surgery and radiotherapy for the primary treatment of small-celled or oat-celled carcinoma of the bronchus. Lancet, ii, 501.

Morrison, R., Deeley, T.J. \& Cleland, W.P. (1963) The treatment of carcinoma of the bronchus. Lancet, $i, 683$. 